\section{Mutations in a new member of the chromodomain gene family cause CHARGE syndrome}

Lisenka E L M Vissers ${ }^{1}$, Conny M A van Ravenswaaij ${ }^{1}$, Ronald Admiraal $^{2}$, Jane A Hurst ${ }^{3}$, Bert B A de Vries ${ }^{1}$, Irene $M$ Janssen ${ }^{1}$, Walter A van der Vliet $^{1}$, Erik H L P G Huys ${ }^{1}$, Pieter J de Jong ${ }^{4}$, Ben C J Hamel ${ }^{1}$, Eric F P M Schoenmakers ${ }^{1}$, Han G Brunner ${ }^{1}$, Joris A Veltman ${ }^{1}$ \& Ad Geurts van Kessel ${ }^{1}$

CHARGE syndrome is a common cause of congenital anomalies affecting several tissues in a nonrandom fashion. We report a 2.3-Mb de novo overlapping microdeletion on chromosome $8 q 12$ identified by array comparative genomic hybridization in two individuals with CHARGE syndrome. Sequence analysis of genes located in this region detected mutations in the gene CHD7 in 10 of 17 individuals with CHARGE syndrome without microdeletions, accounting for the disease in most affected individuals.

CHARGE syndrome is a nonrandom pattern of congenital anomalies including choanal atresia and malformations of the heart, inner ear and retina ${ }^{1}$ (Table 1). With an estimated birth incidence of 1:12,000, CHARGE syndrome is a common cause of congenital anomalies ${ }^{2}$. Most cases of CHARGE syndrome are sporadic, but several aspects of this condition, including the existence of rare familial cases and a high concordance rate in monozygotic twins, support the involvement of a genetic factor. Cytogenetic abnormalities have been described previously, but no specific locus has been identified ${ }^{3}$. Systematic genome scans by conventional comparative genomic hybridization $(\mathrm{CGH})^{4}$ or microsatellite analysis ${ }^{3}$ did not identify a common genetic anomaly; neither did sequencing of candidate genes PAX2 (ref. 5) and PITX2 (ref. 6).

We recently optimized array $\mathrm{CGH}^{7}$ for high-resolution genomewide screening of submicroscopic copy-number changes ${ }^{8}$ and used this approach to identify microdeletions or duplications underlying CHARGE syndrome. We cohybridized genomic DNA from two individuals with CHARGE syndrome with normal reference DNA onto a genome-wide BAC array with $1-\mathrm{Mb}$ resolution (Supplementary Methods online). The genome-wide array CGH profile from one of these individuals is shown in Figure 1a. The only clones reproducibly deleted in this individual map to chromosomal band $8 \mathrm{q} 12$ and encompass a genomic interval of $\sim 5 \mathrm{Mb}$. We confirmed the deletion by fluorescence in situ hybridization (FISH) analysis and proved that it occurred de novo (Supplementary Fig. 1 online). The second individual with CHARGE syndrome included in this pilot study had no microdeletion or microduplication.

To further characterize the deletion in the index individual and to screen additional individuals for abnormalities of chromosome 8 , we established a tiling resolution chromosome 8 array containing 918 overlapping BAC clones. After hybridizing DNA from the index individual onto this array (Fig. 1b), we detected a deletion of 31 overlapping clones spanning a region of $4.8 \mathrm{Mb}$ on 8q12, extending from RP11-44D19 to RP11-274C23 (Supplementary Fig. 1 online).

Notably, an individual with CHARGE syndrome with an apparently balanced chromosome 8 translocation was previously reported 9 . Hybridization of genomic DNA from this person onto the chromosome 8 BAC array detected two microdeletions overlapping with the one that we identified in the index individual (Fig. 1b and Supplementary Fig. 1 online): one encompassing 6 overlapping clones (from RP11-44D19 to RP11-661A3, $\sim 0.8 \mathrm{Mb}$ ) and one encompassing 11 overlapping clones (from RP11-51L11 to RP11-113D4, 1.5 Mb). Between these two deleted regions, 6 clones $(\sim 0.9 \mathrm{Mb})$ showed normal test-over-reference ratios. We used metaphase FISH analysis to confirm the presence of and determine the boundaries of the two distinct microdeletions in this person (Supplementary Fig. 1 online). Although we could not verify de novo occurrence of the microdeletions in this case, the translocation was previously shown to be de novo 9 .

Using data from these two individuals, we defined a shortest region of deletion overlap encompassing $2.3 \mathrm{Mb}$ of genomic sequence on $8 \mathrm{q} 12$ (Fig. 1c). We then screened 17 additional individuals with CHARGE syndrome using the chromosome 8 tiling array and detected no additional chromosome 8 copy-number changes. Next, we sequenced the coding regions and the intron-exon boundaries of all nine annotated or predicted genes located in or just outside the shortest region of deletion overlap (Fig. 1c). We identified ten heterozygous mutations in the gene $\mathrm{CHD}$, including seven stopcodon mutations, two missense mutations and one mutation at an intron-exon boundary (Fig. 1d,e and Table 1). CHD7 consists of 38 exons and has a genomic size of $188 \mathrm{~kb}$. The stop-codon mutations were scattered throughout the gene: two in exon 2, one in exon 3, one in exon 26, two in exon 30 and one in exon 35. The two de novo missense mutations are predicted to lead to the amino acid substitutions I1028V (in exon 12) and L1257R (in exon 15). We observed one de novo mutation $7 \mathrm{bp}$ upstream of exon 26 that could possibly affect splicing (IVS26-7G $\rightarrow$ A).

${ }^{1}$ Departments of Human Genetics and ${ }^{2}$ Otorhinolaryngology, University Medical Center Nijmegen, PO Box 9101,6500 HB Nijmegen, The Netherlands. ${ }^{3}$ Department of Clinical Genetics, The Churchill Hospital, Old Road, Headington, Oxford OX3 7LJ, UK. ${ }^{4}$ Children's Hospital Oakland Research Institute, BACPAC Resources, 74752 nd Street, Oakland, California 94609-1809, USA. Correspondence should be addressed to J.A.V. (j.veltman@antrg.umcn.nl).

Published online 8 August 2004; doi:10.1038/ng1407 


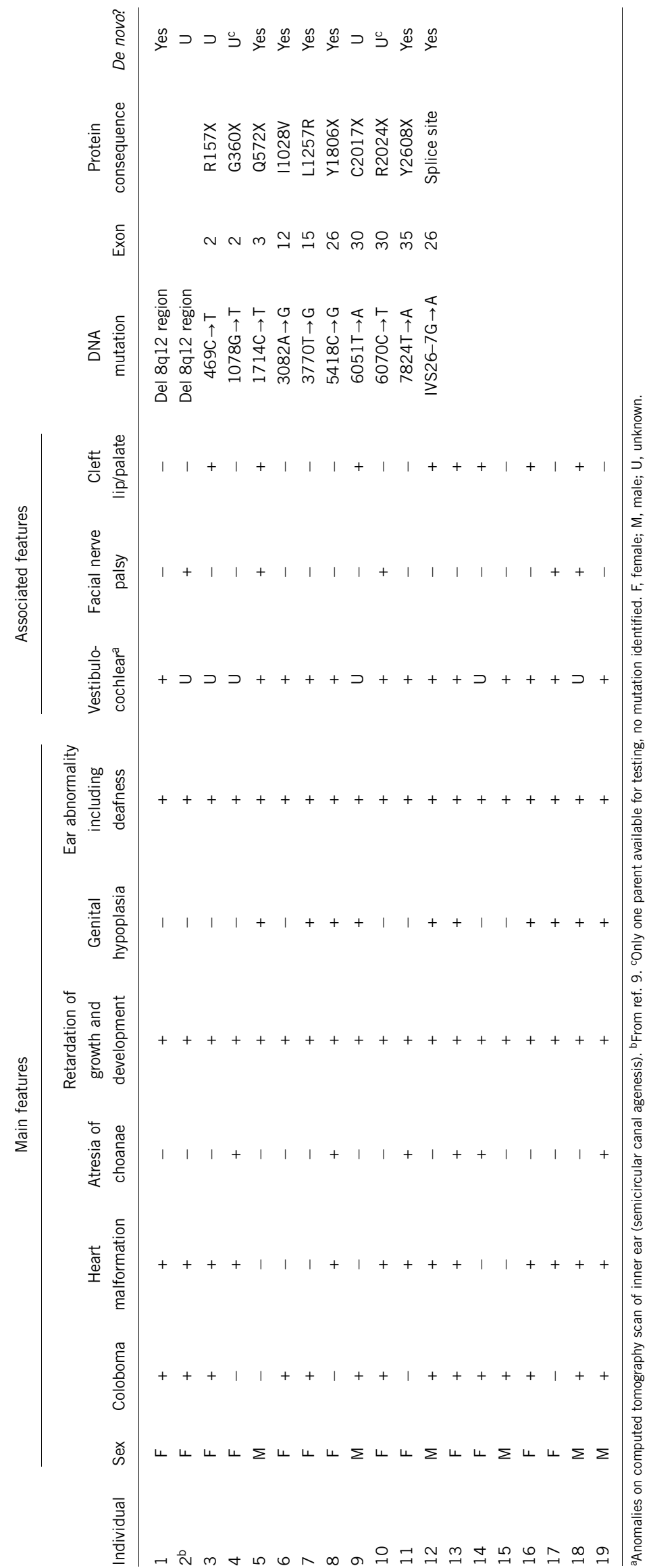

cDNA clone KIAA1416 (ref. 10), encompassing the last 34 exons of $\mathrm{CHD7}$, is a member of the chromodomain helicase DNA-binding (CHD) genes. CHD proteins belong to a superfamily of proteins that have a unique combination of functional domains, including two $\mathrm{N}$-terminal chromodomains, a SNF2-like ATPase/helicase domain and a DNA-binding domain ${ }^{11}$ (Fig. 1d). This class of proteins is thought to have pivotal roles in early embryonic development by affecting chromatin structure and gene expression ${ }^{12}$. The congenital malformations found in CHARGE syndrome have their origin in early embryonic development. CHD7 has ubiquitous expression in several fetal and adult tissues (Supplementary Fig. 2 online), including those affected in CHARGE syndrome.

The identification of seven heterozygous CHD7 stop-codon mutations and two single-copy 8q12 deletions of CHD7 indicate that haploinsufficiency of this gene could account for most cases of CHARGE syndrome. Two amino acid changes are located in one of the functional domains of CHD7, the SNF2 domain, and both affect a conserved amino acid (Supplementary Fig. 3 online). We did not observe any overt phenotypic difference between individuals with $8 \mathrm{q} 12$ deletions and those with nonsense or missense mutations in $\mathrm{CHD} 7$ (Table 1). The index individual, with a $4.8-\mathrm{Mb}$ deletion, has relatively severe mental retardation, which may be due to the deletion of genes adjacent to CHD7. In seven of the individuals with CHARGE syndrome that we studied, we identified no $\mathrm{CHD} 7$ mutations or deletions. This might be due to the presence of intronic or promoter mutations or to whole-exon deletions. CHARGE syndrome might also have a genetically heterogeneous etiology, as different genomic abnormalities have been identified in affected individuals ${ }^{3,4}$. Notably, monosomy with respect to 22q11.2 has been reported to occur in individuals with features of both DiGeorge syndrome and CHARGE syndrome ${ }^{13}$. Deletion of $22 \mathrm{q} 11.2$ was excluded in the individuals in this study who did not have CHD7 deletions or mutations.

Microdeletions encompassing the underlying gene have been reported to occur at low frequencies in single-gene disorders ${ }^{14,15}$. We show that high-resolution genome-wide screening by array CGH is an effective new approach to localize such underlying genes. This approach is of particular interest for sporadic malformation syndromes that cannot be tackled by other mapping approaches because of reproductive lethality. 

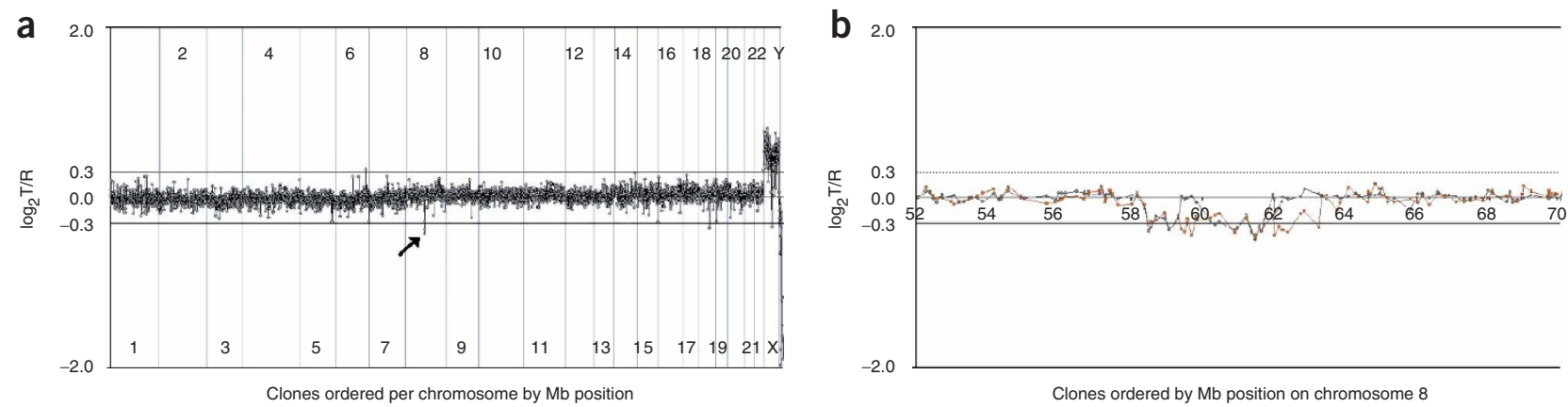

C
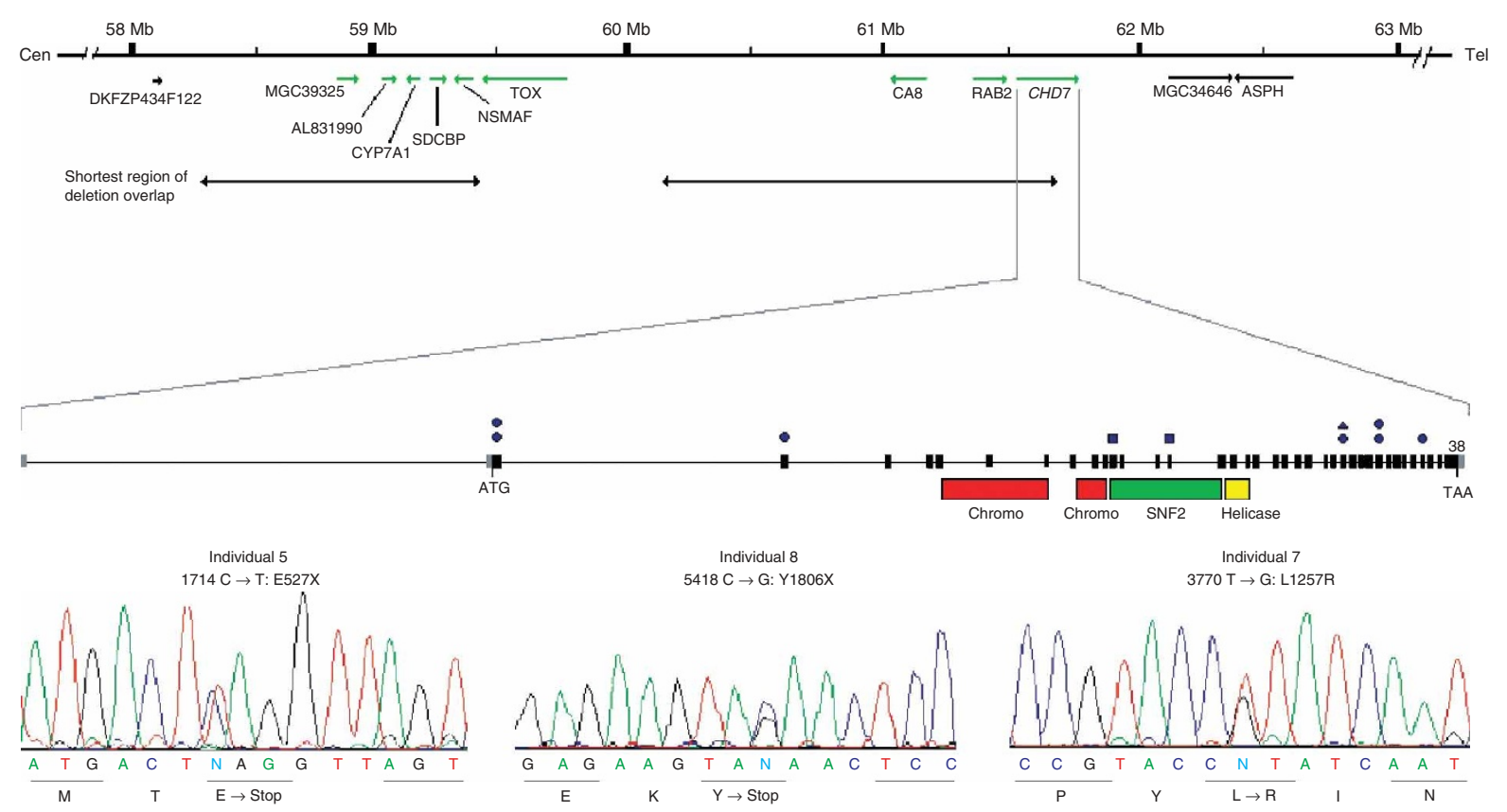

(2) Figure 1 Array CGH profiles of two individuals with CHARGE syndrome, detailed genomic view of $8 \mathrm{q} 12$, organization of $\mathrm{CHD7}$ and mutations detected.

(a) Array CGH genome-wide profile of the index individual with a copy-number deletion of three adjacent clones on 8 q12 (arrow). This profile represents the result of a single hybridization experiment; analysis of the replicate experiment identified only the 8q12 clones as being reproducibly deleted. Vertical lines ndicate chromosome boundaries. (b) Profile of the same individual (brown squares) on the tiling chromosome 8 BAC array with 31 clones characterizing the deletion, and the chromosome 8 profile of an individual (black circles) with an apparently balanced $t(6 ; 8)$ translocation that overlaps with the deletion of the index individual. $(\mathbf{a}, \mathbf{b})$ Clones are ordered on the $x$ axis according to physical mapping positions; $\log _{2}$-transformed test-over-reference (T/R) ratios for each clone are given on the $y$ axis. (c) Transcript map of the deleted $8 q 12$ genomic region. The shortest region of deletion overlap in the two individuals is shown. Genes in green were screened for mutations. Cen, centromeric; Tel, telomeric. (d) Genomic structure of CHD7 indicating the positions of seven nonsense mutations (circles), two missense mutations (squares) and one intron-exon boundary mutation (triangle). The corresponding functional CHD7 domains are marked (colored bars). (e) Partial electropherograms obtained by direct sequencing of PCR products showing two nonsense mutations in individual $5(1714 \mathrm{C} \rightarrow \mathrm{T})$ and individual $8(5418 \mathrm{C} \rightarrow \mathrm{G})$ and one missense mutation in individual $7(3770 \mathrm{~T} \rightarrow \mathrm{G})$.

\section{ACKNOWLEDGMENTS}

We thank the affected individuals and their parents for their participation, H. Straatman and M. Leisink for statistical assistance, E. van Wijk and M. Sweers for technical assistance, E. Bongers for collecting samples from affected individuals, the sequence facility of our department and MACROGEN for sequencing, K. Osoegawa for participation in the production of the microarrays and M. Marra and M. Krzywinski for suggestions relating to the minimal BAC tiling set. This work was supported by grants from the Netherlands Organisation for Health Research and Development (B.B.A.d.V., H.G.B. and J.A.V.).

\section{COMPETING INTERESTS STATEMENT}

The authors declare that they have no competing financial interests.

Received 26 April; accepted 29 June 2004

Published online at http://www.nature.com/naturegenetics/
1. Pagon, R.A. et al. J. Pediatr. 99, 223-227 (1981).

2. Kallen, K. et al. Teratology 60, 334-343 (1999).

3. Lalani, S.R. et al. Am. J. Med. Genet. 118A, 260-266 (2003).

4. Sanlaville, D. et al. Clin. Genet. 61, 135-138 (2002).

5. Tellier, A.L. et al. Am. J. Med. Genet. 93, 85-88 (2000).

6. Martin, D.M. et al. Am. J. Med. Genet. 111, 27-30 (2002).

7. Pinkel, D. et al. Nat. Genet. 20, 207-211 (1998).

8. Vissers, L.E.L.M. et al. Am. J. Hum. Genet. 73, 1261-1270 (2003).

9. Hurst, J.A. et al. J. Med. Genet. 28, 54-55 (1991).

10. Nagase, T. et al. DNA Res. 7, 65-73 (2000).

11. Woodage, T. et al. Proc. Natl. Acad. Sci. USA 94, 11472-11477 (1997).

12. Cavalli, G. et al. Curr. Opin. Cell Biol. 10, 354-360 (1998).

13. de Lonlay-Debeney, P. et al. J. Med. Genet. 34, 986-989 (1997).

14. De Kok, Y.J. et al. Science 267, 685-88 (1995).

15. Johnson, D. et al. Am. J. Hum. Genet. 63, 1282-1293 (1998). 